\title{
BIOMAS: a bio-economic modelling and assessment system for fisheries management strategy evaluation
}

\author{
M.C. Ives $^{\text {a }}$ \\ ${ }^{a}$ Wild Fisheries Research, NSW Department of Primary Industries, PO Box 21, Cronulla, NSW 2230, \\ Australia \\ Email: matthew.ives@industry.nsw.gov.au
}

\begin{abstract}
The primary goal of data collection on exploited fish stocks is to enable the development of credible stock assessment. Ideally, enough information will be collected to enable management strategy evaluations on the stock, providing guidance on future management decisions. In many fisheries management agencies, however, the ability to rapidly and cost-effectively build and run models to evaluate management strategies is as much an impediment to their development as the lack of data.
\end{abstract}

The BIOMAS software provides users with the ability to rapidly develop single or multi-species, lengthbased dynamic population models for the exploration of alternative management strategies in a Bayesianbased framework. The system is theoretically capable of incorporating any number of species, fishing fleets and spatial compartments in a single model. Further complexity is provided with the ability to implement environmental effects on stocks as well as the tracking of economic costs and revenue from fishing activity. However, in practice model complexity will be limited by the needs of the modelling project, by the availability of data and by the modelling project timeline.

By incorporating Bayesian methods including prior probability distributions the BIOMAS system enables users to explore the uncertainty surrounding a limited number of parameter values and to incorporate this uncertainty into the model results. Sensitivity analyses can be run against virtually all model parameters providing insight into the key drivers for the model and likely candidates for Bayesian priors. A number of management indicators are provided for all stocks and fleets in the model including stock depletion, catch weights, age- and length-frequencies, discard mortality estimates and fleet profits - each with Bayesian-based confidence intervals. Such indicators become the primary means of comparing the effectiveness of alternative management strategies when using the system forecasting capabilities. Through the inclusion of stochastic processes, such as process error and environmental and pricing fluctuations, each management strategy can also be evaluated for its robustness to such uncertainty.

The ability to reflect more of our uncertainty in modelled outcomes which tools such as BIOMAS provide can unfortunately come at the expense of clarity in the outcomes. This is only because these models better encompass our lack of knowledge about fishery systems. Fortunately, such modelling tools can also provide us with a clearer understanding of the main sources of this uncertainty. Furthermore, the unique design of the BIOMAS system allows users to also undertake 'research strategy evaluations' in which the costs and benefits of different forms of research are compared, allowing managers and research leaders to see more clearly the benefits of targeted research to reduce these uncertainties.

Keywords: Population modelling; management strategy evaluation; fisheries management; research strategy evaluations; uncertainty 


\section{INTRODUCTION}

The need to better understand the population dynamics of fish stocks and their interactions with human systems has given rise to the modelling methodology known as Management Strategy Evaluation (MSE), also known as "Harvest Strategy Evaluation", "Operating Management Procedures" or "Management Procedure Evaluation" (Sainsbury et al., 2000; Scandol, 2004). An MSE involves a decision analysis comparing the expected consequences of alternative actions across a set of well-defined alternative hypotheses about the future states of the system being modelled. Thus, a model structure with certain parameter values (which together represent a hypothesis) is projected into the future under a range of scenarios in order to examine and compare the outcomes of alternative management strategies. Uncertainty is introduced through the addition of stochastic processes and through the inclusion of prior knowledge (and uncertainty) about model parameters using Bayesian inference (Punt and Hilborn, 1997; McAllister and Kirkwood, 1998; Chen et al., 2003).

Ideally an MSE framework should include all of the major components of a fisheries management scenario including: 1) the natural systems and fish stocks (operating model); 2) the exploitative commercial and recreational fisheries; 3) the collection and analysis of data by research programs; 4) the socio-economic system that supplies inputs and dictates costs and prices; and, 5) the fisheries management/political systems that imposes management actions on the fishery (Figure 1). As shown in Figure 1 there is a high degree of interaction between each of these systems and because each of these components are in themselves highly complex, the extent to which they are modelled in some MSE's can be quite limited. Generally the majority of the modelling effort is focused on the biological system (operating model) for which there is a longer history of development in the field of fisheries stock assessment.

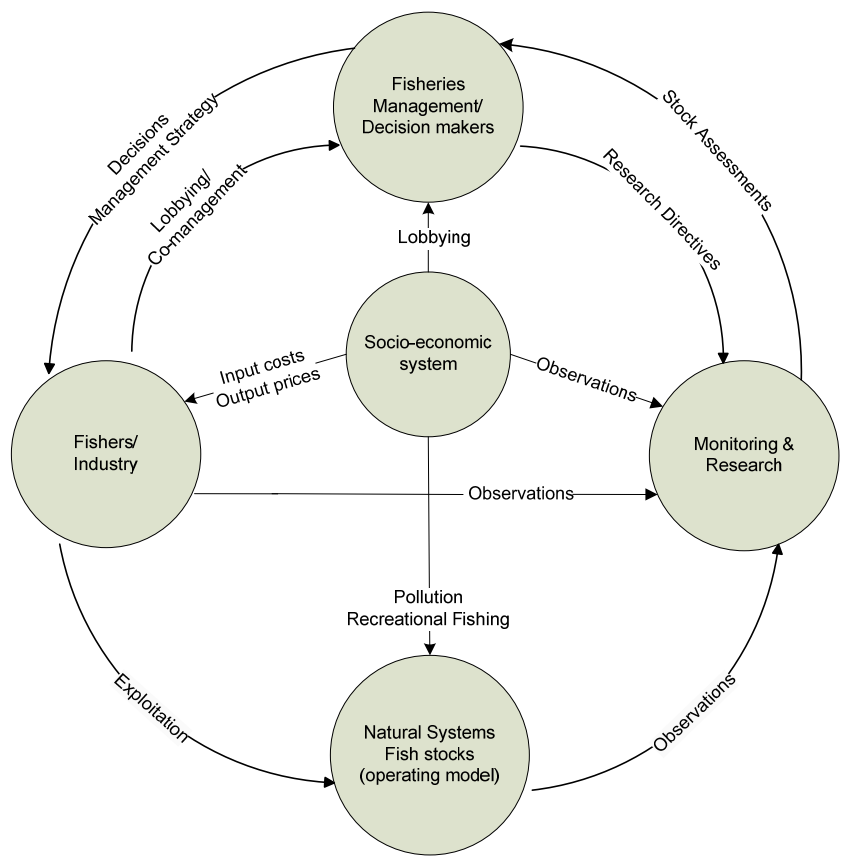

Figure 1: A conceptual representation of the main components (systems) modelled within the management strategy framework. Adapted from (Lane and Stephenson, 1998)
In many fisheries management agencies the ability to rapidly and cost-effectively build and run such MSE models is as much an hindrance to their use as is the lack of sufficient data. The BIOMAS software package provides users with the ability to rapidly develop such models for the exploration of alternative management strategies in a Bayesian-based framework. BIOMAS was developed primarily as an assessment tool for single or multiplestocks and contains components that represent each of the main MSE components shown in Figure 1.

This manuscript provides a brief description of the BIOMAS design with some discussion on the system's uses, as well as its strengths and limitations. For case studies on the application of BIOMAS to actual fisheries as well as a presentation of many of the equations that make up the various components of BIOMAS, readers are directed to (Ives and Scandol, 2007; Ives et al., 2009).

\section{SYSTEM DESIGN}

The BIOMAS system includes in its design a number of important programming devices, the most important being the application of object-oriented programming (OOP) (Ferreira, 1995; Groot and Lantinga, 2004) which has enabled a high degree of flexibility in model configuration. The basic concept of OOP is to view the things being modelled as objects, which interact in pre-defined ways with other objects. By building the model in this way it becomes theoretically capable of incorporating any number of objects such as species, fishing fleets and spatial areas in virtually any possible configuration. This flexibility inherent in the BIOMAS design extends to the user interface that allows users to rapidly build and manage all such modelling scenarios and extends to the model outputs which include an extensive number of output files of parameter values and management indicators for all of the possible fleet, species and spatial configurations. 
In practice, the availability of data and modelling time will put practical limits on the complexity of the model.

\section{BIOLOGICAL OPERATING MODEL}

The underlying biological operating model is a stage/size-structured dynamic meta-population model with each species stock occupying one or more areas as discrete subpopulations within each area. Such a design allows for each species to have species-wide properties while each separate meta-population of a species is subject to differing environmental and anthropomorphic influences in their respective areas. Figure 2 contains a simple model design example with two areas that contain two separate sub-populations of a single species.

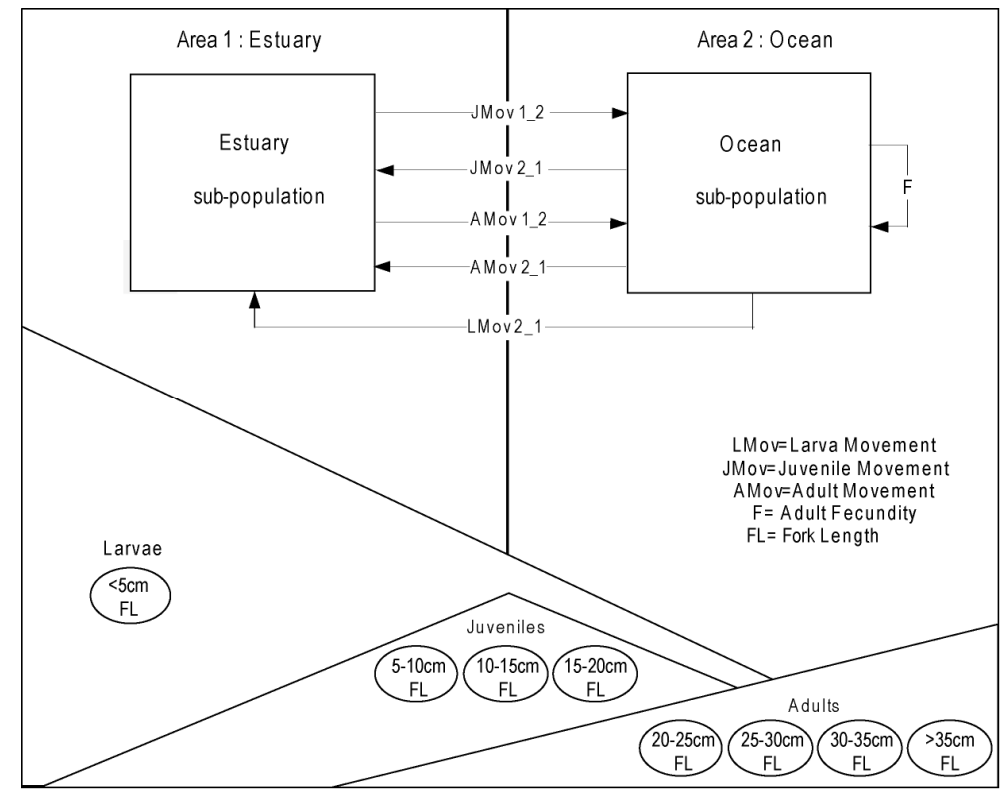

Figure 2: An example of a stage-size structured meta-population model built using BIOMAS. The upper area displays the two sub-populations within each area and shows the movement between these sub-populations. The lower area displays the various life stages based on fork lengths (FL) into which individuals are graded. The polygons provide a stylistic representation of the relative numbers of individuals of the three main life stages - larva, juvenile and adult - and in which area these life stages predominantly reside.

Rather than implementing a fully individual-based model, BIOMAS instead aggregates individuals into groups or stages (Walters and Martell, 2004, Box 5.3). Thus, each individual fish exists only as one of a number of fish contained in a length stage, which is in turn contained in a life stage (e.g. larva, juvenile, adult). By grouping individuals in this manner the model computations are reduced, as are the number of parameters required to run the model. For example, as shown in Figure 2, movement parameters are applied to life stages rather than length stages thereby enabling flexibility in the movement of various life stages while minimising the number of parameters required. This stage-based methodology is applied throughout the model structure with individuals grouped into different sexes, lengths, ages, life stages, growth paths and price grades. The stages are completely configurable so the user can choose to include any number of length, sex, age or life stages for a particular species according to the needs of their modelling exercise.

A series of parameters relevant to fish species characteristics are used to configure the model population dynamics for each species, and to some extent the model structure. As shown in Figure 3 these processes are broken into growth, recruitment, movement (immigration and emigration), natural mortality and fishing mortality. Growth is based on a von Bertalanffy growth function with variability in growth incorporated through the use of a series of five aggregate growth paths into which individuals are grouped (Punt et al., 2001). Given the importance of recruitment in population dynamics the BIOMAS system provides users with a choice between a small number of alternative stock-recruitment relationships (including the Beverton-Holt function (Beverton and Holt, 1957)) as well as a customisable fecundity to length relationship. Movement is based on configurable probabilities of movement of each life stage from one area to another. Natural mortality consists of an instant mortality rate for each life stage which allows for higher early-life mortality. Fishing mortality is driven by actual effort during the calibration period and by simulated fleet dynamics during the forecast periods (discussed in more detail below). Also included in each of these components are a number of configurable stochastic processes such as recruitment error (process error), observation error, implementation error and other stochastic environmental and economic processes. 


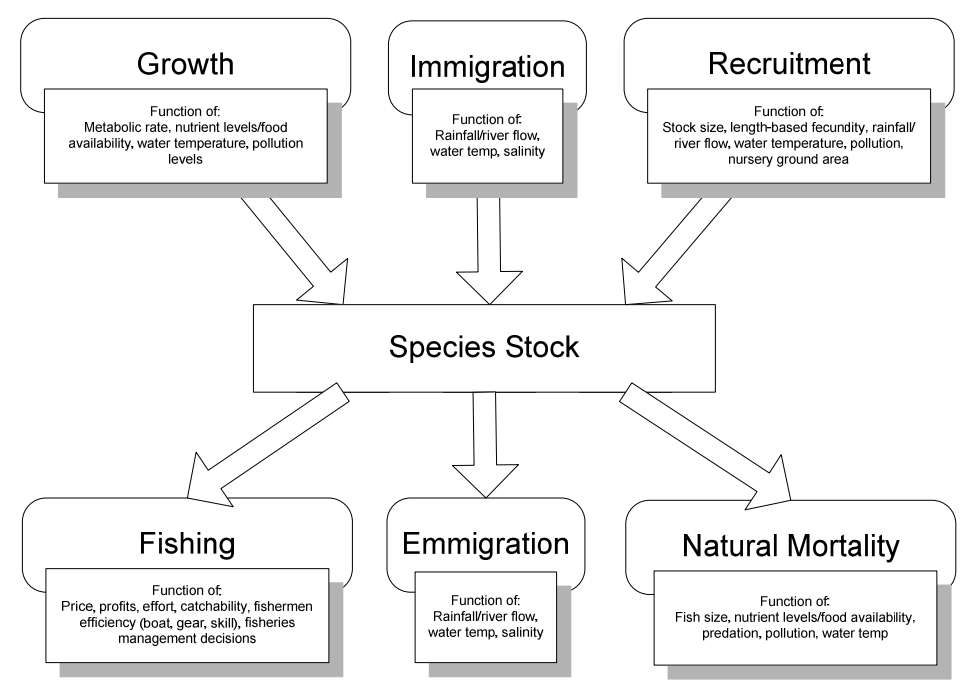

Figure 3: Basic population dynamics within the operating model showing configurable interactions with environmental and socio-economic/management effects

A unique feature of the BIOMAS system is the ability to apply effects from environmental and management components to species and fishing fleet processes (as shown in Figure 3). BIOMAS was originally developed to produce models capable of examining the possible effects of climate change on fisheries dynamics. Through the use of object-oriented programming (OOP) the effects component was built to allow any environmental component to affect any other environmental component, as well as any species, fleet or economic component. Management strategies are also implemented using this same design so that management actions consist basically of research and monitoring processes triggering effects on the dynamics of commercial and recreational fleets.

\section{FISHERS}

In the BIOMAS design fishing is conducted by fishing fleets rather than individual fishers. Each fleet has a single fishing method with a configurable gear selectivity function and a single unit of effort. Catch is based on a Baranov catch equation with fishing mortality tied to effort through a catchability (or fleet efficiency) parameter. Effort within an area is assigned to the fleet as a whole and the catch of the whole fleet is pooled for each species. The effort of the fleet is thus best described as the standardised effort of the average fleet vessel. However, the effort units are completely flexible and need not be in fishing days but could instead be expressed in terms of kilowatt days in order to capture the effect of engine power on gear efficiency or catchability (Eigaard et al., 2011).

Future projections of fishing effort require a certain level of complexity to provide realistic reactions of fishers to alternative management strategies. Through the use of management effects a BIOMAS model is able to apply a large range of hypotheses regarding fisher dynamics, such as fleet effort tied to the catch-perunit-effort of a particular species (e.g. Ives et al., 2009), product prices, or costs, or a combination of any such effects. The system also permits users to include knowledge of past effort levels by applying a percentage of the effort from the average of a configurable number of past years.

\section{MONITORING AND RESEARCH}

The monitoring and research component gathers information from the fishery and produces calculated indicators for future management and monitoring actions. As shown in Error! Reference source not found. research sampling occurs each month prior to the pre-fishing management actions. Data is either gathered from sampling using an existing fishing fleet or from a fishery-independent monitoring fleet with its own gear and sampling regime. The data gathered from each fleet includes monthly catch, discard and estimated discard mortality weight as well as monthly length- and age-frequency samples. All such data contain a configurable amount of observation error. Once collected the data is then used to calculate a series of leading parameters or management indicators (Forrest et al., 2008) for each species including average weight, estimated numbers and biomass, biomass depletion ratio and spawning potential ratio, as well as indicators for each fleet including average annual catch, catch-per-unit-effort and a number of economic indicators (described below). 


\section{ECONOMICS}

The economic component in the current release of BIOMAS (2.0) is fairly rudimentary and involves simply applying prices to all fisher inputs and outputs with the inclusion of a discount rate for calculating net present values. Costs are broken into sunk, fixed and variable costs (e.g. (Holland, 2011)) that allow for costs to be associated with each unit of effort (variable costs) as well as to no effort (sunk and fixed costs). Revenue is calculated as the quantity of fish caught multiplied by their price, with configurable price grades tied to one or more length stages. Using this information BIOMAS also calculates a number of key economic indicators for each fleet including average annual costs and revenue, net present discounted profits and average annual profit variability As with all management indicators these can be included in the effects component to allow for more sophisticated fisher dynamic scenarios where effort is tied to past or present revenue, profits or costs.

\section{MODELLING PERIODS}

Each model run undergoes three main time periods. A model run always begins with an initial population of each species that needs to be built up. During this 'burn-in' period each population grows until it achieves a stable virgin state with all age classes represented in their respective areas. The length of the burn-in period required will depend on the maximum age and fecundity of each of the modelled species. Following this the model moves into a period of observed fishing effort and environmental data. All or some part of this time period can be used to calibrate the model. BIOMAS enables the user to calibrate a single model against up to four observed data series using maximum likelihood with configurable weightings. Finally, the model can be run through a forecast period where the best fitting model runs are projected into the future with fishing effort and environmental states simulated based on effect dynamics and historic data. It is during this period that alternative management strategies can be applied and the results of several such strategies compared based on various management indicators.

\section{MODELLING MODES}

There are four main modelling modes within BIOMAS, namely basic simulation, sensitivity analysis, Bayesian simulation and MSE. The basic simulation mode can be used to help build and calibrate a model structure to fixed parameter values. Users can also run forecasts using the basic simulation applying only a single management strategy. The sensitivity analysis mode is used to vary all or some of these model parameters in order to examine the effect each parameter has on the chosen management indicators. The Bayesian simulation mode involves the application of prior probability distributions to a limited number of parameters to provide a posterior distribution for each management indicators that better reflects the prior knowledge (and uncertainty) regarding these input parameters. Finally, in the MSE or forecasting mode the model simulations or Bayesian posterior distributions are projected into the future under the influence of one or more alternative management scenarios.

These four modes can be run individually or used as a series of steps that will help develop a more robust and defensible model. The simulation mode enables the construction of a model that is capable of providing an assessment of the status of the modelled stocks. Most of the work in developing the best model structure is undertaken in this mode. The Bayesian simulation provides similar capabilities as the simulation mode but will also allow the user to include prior knowledge as well as to explore the uncertainty around key model parameters. The sensitivity analysis can be used to inform the simulation modes as to which parameters are the main drivers of the model outputs and which parameters may be the best candidates for Bayesian prior probability distributions. The sensitivity analysis can also help determine whether additional processes (effects) have a significant enough impact on the model to justify their inclusion. Finally, the MSE mode involves the application of a scenario to future projections under a series of management strategies including the status quo. This mode allows an examination of the impact of the alternative management strategies on the chosen management indicators, and their robustness to uncertainty (Lane and Stephenson, 1998).

\section{FISHERIES MANAGEMENT AND MANAGEMENT STRATEGY EVALUATIONS}

The fisheries management component is based on the application of management strategies. Each strategy consists of a number of management actions, which in turn contain one or more management effects. Management effects, as explained above, involve research monitoring processes triggering effects on the dynamics of research, commercial and recreational fleets. An MSE analysis will consist of running two or more such management strategies against the future projection of a chosen calibrated model and comparing the values of pre-determined management indicators. In the case of a basic simulation each strategy will be run for a number of iterations (or trials) with each of the iterations containing a different instance of each of the model's stochastic processes. The range of values produced for each management indicator can then be 
used to compare the relative merits of each management strategy in terms of fishery reference points, as well as in terms of their robustness to the uncertainty represented by the stochastic processes. The Bayesian simulation takes this process one step further by also incorporating the knowledge (and uncertainty) in prior probability distributions of key model parameters into the future projections, giving the results a more complete representation of the knowledge (and uncertainty) in the modelled system.

Deciding between alternative management decisions need not necessarily involve unconditional predictions of the future. It may be sufficient to simply determine which decisions are more likely to perform better under a wide range of possible future scenarios (Walters and Martell, 2004). The key task therefore becomes clearly defining up-front the concrete objectives and constraints that must be satisfied by each alternative strategy (Lane and Stephenson, 1998).

\section{MODEL OUTPUTS}

The model outputs provided by BIOMAS are dependent on the modelling mode but consist primarily of a series of comma-delimited text files (easily opened in Microsoft Excel). A basic single iteration simulation run will produce a series of output files for each species, fleet and area showing the values of all species and fleet parameters, environmental conditions and management indicators for each time step within the model. Also included are the stock weight for each species by area; the number of each species by length in each area; and age and length frequency distributions for the catch and discards of each species for each fleet. A sensitivity analysis will produce a summary of the chosen management indicators with details on the parameter values that were changed in each iteration. A multi-iteration basic simulation or Bayesian simulation run will also produce a summary of the chosen management indicators as well as provide likelihood estimates and all the aforementioned outputs of a single basic simulation for the model run that produced the best likelihood score.

\section{DISCUSSION}

The BIOMAS system provides the user with the ability to efficiently develop bio-economic fisheries models. BIOMAS is designed to run simple single species, single-fleet, non-spatial models or multi-species, multifleet, meta-population models with environmental and economic drivers. Users are provided with a series of standard fisheries modelling parameters with which to calibrate the model and given additional flexibility for non-standard model interactions through the effects component. Each model run provides a large selection of outputs and the four modelling modes enable users to proceed through logical steps from a single basic simulation for simple stock assessment work to a full Bayesian Management Strategy Evaluation.

Despite its power and flexibility the BIOMAS system has its limitations. Firstly, the added flexibility provides by BIOMAS can be both a blessing and a curse. BIOMAS will enable users to more easily explore alternative model structures. However, such flexibility can open users to the unsettling existence of significant (and often ignored) model structure uncertainty (Ives and Scandol, 2007). Secondly, BIOMAS was built primarily as an assessment tool for the management of single or multi-species fisheries and is not designed for ecosystem-based modelling (Smith et al., 2007). The biological system contains no inherent system-based rules (such as the mass balance master equation of Ecopath (Pauly et al., 2000)) to manage interactions between species such as predator-prey dynamics. It is possible to simulate such inter-species interactions using the effects component. However, BIOMAS was not designed for this purpose. It was designed for undertaking stock assessments and MSE's on a commercially important species and is best suited for these tasks.

The BIOMAS system allows users to incorporate stochastic processes in fish population dynamics, environmental components and even economic components, as well as include observation and implementation errors. The addition of such stochastic processes, errors and the use of Bayesian priors can provide modellers with the ability to more fully represent the true uncertainty in their models (Punt and Hilborn, 1997; McAllister and Kirkwood, 1998). However, the ability to reflect more of our uncertainty in modelled outcomes can come at the expense of clarity in the outcomes. (Holland et al., 2005; Ives et al., 2009; Jiao et al., 2009; Campbell et al., 2010), especially if model structure uncertainty is also included (Ives and Scandol, 2007). This is only because the models are more encompassing of our uncertain knowledge. In reality, fisheries systems incorporate highly complex ecosystems containing vast numbers of organisms with extensive connectivity and interactions coupled with equally complex social, economic and political systems that can change dramatically over time. As such, there are few areas of management that contain such deep levels of uncertainty as natural resource management (De Young, 1999). This situation implies that the fisheries management community should be cautious when interpreting the results of complex models if their 
Ives. M, BIOMAS: a bio-economic modelling and assessment system for fisheries management strategy evaluation

additional complexity has come at the expense of providing a more thorough representation of uncertainty (Longhurst, 2006).

Managing the multiple, sometimes competing biological and economic objectives of stakeholders is a problem that fisheries managers have struggled with for many decades (Lane and Stephenson, 1998). The increased capabilities provided by modelling packages such as BIOMAS should help improve our understanding of the complexities involved in fisheries management. However, the ability to model such complexity may not necessarily solve this underlying problem, such as in cases where the uncertainty in the modelled outcomes swamps any differences between management strategies. Fortunately, such modelling efforts also provide us with a clearer understanding of the main sources of these uncertainties. Furthermore, the unique design of the BIOMAS system allows users to also undertake 'research strategy evaluations' in which the costs and benefits of different forms of research are compared. By helping managers and research leaders to see the benefits of more targeted research efforts this relatively new branch of modelling can possibly help reduce the uncertainties that are clouding the results.

Beverton, R.J.H. and Holt, S.J., 1957. On the dynamics of exploited fish populations. Fishery Investigations Series II, 19:1-533.

Campbell, M., Campbell, A., Officer, R.A., O’Neill, M.F., Mayer, D.G., Thwaites, A., Jebreen, E.J., Courtney, A.J., Gribble, N., Lawrence, M.L., Prosser, A.J. and Drabsch, S.L., 2010. Harvest Strategy Evaluation to Optimise the Sustainability and Value of the Queensland Scallop Fishery, Final Report 2006/024. Queensland Government - Primary Industries \& Fisheries, Brisbane.

Chen, Y., Jiao, Y. and Chen, L., 2003. Developing robust frequentist and Bayesian fish stock assessment methods. Fish and Fisheries, 4:105-120.

De Young, B., 1999. Canadian marine fisheries in a changing and uncertain world: a report prepared for the Canadian Global Change Program of the Royal Society of Canada, NRC Research Press.

Eigaard, O.R., Rihan, D., Graham, N., Sala, A. and Zachariassen, K., 2011. Improving fishing effort descriptors: Modelling engine power and gear-size relations of five European trawl fleets. Fisheries Research, 110:39-46.

Ferreira, J.G., 1995. ECOWIN - an object-oriented ecological model for aquatic ecosystems. Ecological Modelling, 79:21-34

Forrest, R.E., Martell, S.J.D., Melnychuk, M.C. and Walters, C.J., 2008. An age-structured model with leading management parameters, incorporating age-specific selectivity and maturity. Canadian Journal of Fisheries and Aquatic Sciences, 65:286-296.

Groot, J.C.J. and Lantinga, E.A., 2004. An object-oriented model of the morphological development and digestibility of perennial ryegrass. Ecological Modelling, 177:297-312.

Holland, D.S., 2011. Planning for changing productivity and catchability in the Maine lobster fishery. Fisheries Research, 110:47-58.

Holland, D.S., Bentley, N. and Lallemand, P., 2005. A bioeconomic analysis of management strategies for rebuilding and maintenance of the NSS rock lobster (Jasus edwardsii) stock in southern New Zealand. Canadian Journal of Fisheries and Aquatic Sciences, 62:1553-1569.

Ives, M.C. and Scandol, J.P., 2007. A Bayesian analysis of NSW eastern king prawn stocks (Melicertus plebejus) using multiple model structures. Fisheries Research, 84:314-327.

Ives, M.C., Scandol, J.P., Montgomery, S.S. and Suthers, I.M., 2009. Modelling the possible effects of climate change on an Australian multi-fleet prawn fishery. Marine and Freshwater Research, 60:1211-1222.

Jiao, Y., Reid, K. and Smith, E., 2009. Model selection uncertainty and Bayesian model averaging in fisheries recruitment modeling. In: R.J. Beamish and B.J. Rothschild (Editor), The Future of Fisheries Science in North America. Springer, New York, pp. 49-76.

Lane, D.E. and Stephenson, R.L., 1998. A framework for risk analysis in fisheries decision-making. ICES J. Mar. Sci., 55:1-13.

Longhurst, A., 2006. The sustainability myth. Fisheries Research, 81:107-112.

McAllister, M.K. and Kirkwood, G.P., 1998. Bayesian stock assessment: a review and example application using the logistic model. ICES Journal of Marine Science, 55:1031-1060.

Pauly, D., Christensen, V. and Walters, C., 2000. Ecopath, Ecosim, and Ecospace as tools for evaluating ecosystem impact of fisheries. ICES Journal of Marine Science: Journal du Conseil, 57:697-706.

Punt, A.E., Campbell, R.A. and Smith, A.D.M., 2001. Evaluating empirical indicators and reference points for fisheries management: application to the broadbill swordfish fishery off eastern Australia. Marine and Freshwater Research, 52:819-832.

Punt, A.E. and Hilborn, R., 1997. Fisheries stock assessment and decision analysis: the Bayesian approach. Reviews in Fish Biology and Fisheries, 7:35-63.

Sainsbury, K.J., Punt, A.E. and Smith, A.D.M., 2000. Design of operational management strategies for achieving fishery ecosystem objectives. ICES J. Mar. Sci., 57:731-741.

Scandol, J.P., 2004. A Framework for the Assessment of Harvested Fish Resources in NSW, NSW Department of Primary Industries, Cronulla.

Smith, A.D.M., Fulton, E.J., Hobday, A.J., Smith, D.C. and Shoulder, P., 2007. Scientific tools to support the practical implementation of ecosystem-based fisheries management. ICES Journal of Marine Science: Journal du Conseil, 64:633639.

Walters, C. and Martell, J.D., 2004. Fisheries Ecology and Management. Princeton University Press, New Jersey. 